\title{
IDENTIFICACIÓN DEL AGENTE CAUSANTE DE LA MARCHITEZ DE PROTEAS (Leucadendron sp.)
}

\section{IDENTIFICATION OF THE CAUSAL AGENT OF THE WILTING OF PROTEAS (Leucadendron sp.)}

\author{
${ }^{1}$ Ingrid Carolina Salazar, ${ }^{2}$ Edgar Martínez Granja, ${ }^{3}$ Fernando Peña, ${ }^{4}$ Marco Cabezas, ${ }^{5} J o h n$ Gómez
}

${ }^{1}$ I.A. Correo electrónico: dirgni_c@msn.com ${ }^{2}$ I.A., M.Sc., Ph.D., Docente investigador, Ingeniería Agronómica, U.D.C.A. Correo electrónico: edgar.martinezgranja@gmail.com. ${ }^{3}$ I.A., M.Sc, Docente investigador, Ingeniería Agronómica, U.D.C.A. Correo electrónico: ingagro@udca.edu.co. ${ }^{4}$ I.A. MSc, c.Ph.D. Docente investigador, Ingeniería Agronómica, U.D.C.A. Correo electrónico: mcabezas@udca.edu.co. ${ }^{5}$ I.A., M.Sc., Docente investigador, Ingeniería Agronómica, U.D.C.A. Correo electrónico: ingagro@udca.edu.co.

Rev. U.D.C.A Act. E Div. Cient. 13 (2): 79-87, 2010

RESUMEN

Las proteas son de reciente establecimiento en el departamento de Cundinamarca, donde se han realizado proyectos empresariales de producción del género Leucadendron, cultivo que ha presentado la enfermedad denominada marchitez. Para identificar su agente causal, se llevó a cabo una investigación utilizando métodos convencionales de diagnóstico, para aislar el agente causal y comprobar su patogenicidad, en el laboratorio; además, se efectuó su caracterización molecular, mediante la extracción y la amplificación del ADN. El fragmento amplificado, se secuenció empleando BigDye ${ }^{\circledR}$ Terminator v3.1 Cycle Sequencing Kit, según las especificaciones del manufacturero, en un secuenciador ABI3730XL (Applied Biosystems). En laboratorio, se evaluó la patogenicidad y el efecto de los dos fitopatógenos aislados sobre la incidencia de la enfermedad, en las variedades de proteas Gold Strike y Petra. Las cepas de los hongos, se inocularon individualmente y, en mezcla, aplicando un diseño completamente al azar, con arreglo factorial $2 \times 4$. En las inoculaciones, se empleó una suspensión de $10^{5} \mathrm{~mL}^{-1}$ macroconidias; también se evaluaron en el campo, en igual concentración y sobre las mismas variedades, empleando un diseño de bloques completamente al azar, con arreglo factorial $2 \times 4$. En el diagnóstico de laboratorio, se encontraron dos cepas de Fusarium spp., diferenciadas por el color de la colonia. Éstas correspondieron, en el análisis molecular, a dos especies: F. oxysporum (colonia blanca) y Fusarium sp. (colonia roja). Se confirmó la patogenicidad de las dos especies en las variedades evaluadas.

Palabras clave: Hongo, Fusarium, Petra, Gold Strike, floricultura.

\section{SUMMARY}

Plantations of proteas are new at the department of Cundinamarca, where businesses of production of Leucadendron have been carried out. In these plantations a new disease denominated wilting has been observed. To identify the causal agent of this disease conventional plant pathological tests were conducted to isolate and to verify the pathogenicity of the causal agent under laboratory conditions. Also a molecular characterization was conducted through extraction and amplification of DNA. The DNA amplified fragment was sequenced by BigDye ${ }^{\circledR}$ Terminator v3.1 Cycle Sequencing Kit according to the manufacturer specifications in a sequencer ABI3730XL (Applied Biosystems). Under laboratory conditions the pathogenicity and disease incidence caused by two isolates of fungi obtained were evaluated on the varieties Gold Strike and Petra. The fungi were inoculated both individually and in mixture, using a completely randomized design with a factorial arrangement of $2 \times 4$. In the inoculations was used a suspension of $10^{5}$ macroconidia $\mathrm{mL}^{-1}$ of water. Also they were evaluated under field conditions at the same concentration and on the same varieties, using a complete block design with factorial arrangement of $2 \times 4$. The laboratory analysis indicated the presence of two species of Fusarium, which were differentiated through the color of their colonies. According to the molecular analysis, they belong to F. oxysporum (white colony) and Fusarium sp. (red colony). It was confirmed the pathogenicity of both species on the tested varieties.

Key words: Fungus, Fusarium, Petra, Gold Strike, floriculture. 


\section{INTRODUCCIÓN}

En algunos municipios de Cundinamarca, se inició hace tres años, aproximadamente, la siembra de proteas del género Leucadendron. En la actualidad, alcanza un área aproximada de 35ha. Este género pertenece a la familia Proteaceae, la cual, es originaria de Suráfrica y de Australia (Rabelo 1995; Salinger, 1991). En Suráfrica existen cerca de 400 especies, que constituyen el grupo más promisorio, con un alto potencial para su utilización, como flores de corte (Malan, 1992). Su cultivo y la tecnología para su producción, se inició en Israel, hace 30 años (Ben, 1986). El género Leucadendron, en particular, se considera como el más cultivado y fuerte en el mercado de exportación, como flor de corte (Barkera et al. 2004; Matthews, 2002).

En el género Leucadendron, se destacan las variedades Petra y Gold Strike, que fueron desarrolladas, específicamente, como acompañantes verdes; poseen tallos largos que exceden los $60 \mathrm{~cm}$; sus flores conservan la calidad por más de 60 días en el florero, sin presentar síntomas de marchitez (ISHS, 2007). En Colombia es un cultivo nuevo y no se ha generado información técnica local relacionada con los factores que favorecen y limitan su producción. Entre los limitantes fitosanitarios está la marchitez, que es una enfermedad que preocupa a los productores. En muestras de plantas enfermas, analizadas en el Laboratorio de Sanidad Vegetal de la Universidad de Ciencias Aplicadas y Ambientales U.D.C.A, se observaron síntomas característicos de un marchitamiento generalizado de la planta: pérdida de foliolos, reducción del crecimiento y secamiento progresivo. Los tejidos internos de tallos afectados presentan una coloración marrón, cuya longitud aumenta a través del tiempo y se extiende desde las raíces hasta la parte aérea. Se afectan los haces vasculares del floema y del xilema, que conlleva clorosis, muerte de nuevos brotes y de la planta (Lubbe et al. 2008).

Existen varios fitopatógenos de gran importancia, que afectan al género Leucadendron: Phytophthora cinnamomi, Rhizoctonia spp., Fusarium oxysporum, (Moura \& Rodrigues, 2001; Denman, 2001; Crous et al. 2004); Alternaria sp., Cladosporium sp. y Botrytis sp. (Crous \& Palm, 1999).

Según Dunne et al. (2003), en Australia existen tres especies de hongos fitopatógenos asociadas con la marchitez en Leucadendron: Fusarium oxysporum, F. solani y F. moniliforme. Se destaca la especie $F$. oxysporum, porque es ampliamente distribuida en diferentes regiones del mundo, tiene una amplia gama de hospedantes y formae specialis y es económicamente importante, como agente causal de la fusariosis (Taylor $\&$ Crous, 2000; Leslie $\mathcal{E}$ Summerell, 2006). Estos fitopatógenos son habitantes del suelo, están ampliamente distribuidos en el trópico y causan marchitamientos vasculares sistémicos, que son de importancia económica (Crous et al. 2004; Leslie $\mathcal{E}$ Summerell, 2006). La importancia de la marchitez vascular es a menudo subestimada, porque el desarrollo de los síntomas es lento y, rara vez, produce muerte de la planta (Lubbe et al. 2008). Después de la infección, los brotes mueren y se presenta una reducción en el número de flores producidas por planta (Swart et al. 2001). En Colombia, se han observado síntomas de marchitez en las variedades Petra y Gold Strike, en diferentes cultivos de proteas, establecidos en el departamento de Cundinamarca.

La enfermedad se disemina, principalmente, por los tallos infectados que se utilizan para siembra, los cuales, han dado origen a plantas con marchitez y con síntomas vasculares, algunas semanas después del trasplante del material vegetal, proveniente de centros de propagación. En estas plantas sintomáticas, se ha detectado la presencia del género Fusarium (Herrera et al. 2006). En Chile, se realizaron pruebas de control químico y biológico y se determinó que el fungicida Benomil y el hongo Trichoderma harzianum, en las concentraciones $10^{8}$ y $10^{9}$ conidias $\mathrm{mL}^{-1}$, fueron efectivos en el control preventivo de Fusarium sp. (Obreque, 2004; Herrera et al. 2006; Crous et al. 2004).

Debido al desconocimiento del agente causal de la enfermedad, presente en el material vegetal de propagación de origen ecuatoriano y a la incidencia de Fusarium oxysporum en suelos dedicados a la floricultura en la Sabana de Bogotá, se requiere profundizar en el estudio de la marchitez y en el entendimiento de los factores que influyen en su presencia en el cultivo. El objetivo de esta investigación fue identificar al agente causal de la marchitez en proteas del género Leucadendron y determinar su incidencia y su severidad, en condiciones de cultivo.

\section{MATERIALES Y MÉTODOS}

El presente trabajo, se llevó a cabo en el Laboratorio de Sanidad Vegetal y en la sede experimental El Remanso, de la Facultad de Ingeniería Agronómica, de la Universidad de Ciencias Aplicadas y Ambientales U.D.C.A, localizada a $4^{\circ} 47^{\prime}$ $57^{\prime \prime}$ Norte y $74^{\circ} 02^{\prime} 40^{\prime \prime}$ Oeste, a $2.560 \mathrm{msnm}$, temperatura media de $14,5^{\circ} \mathrm{C}$ y $860 \mathrm{~mm}$ de precipitación media anual en régimen bimodal.

Para la identificación del agente causal de la enfermedad, se recolectó material vegetal de proteas del género Leucadendron, en cultivos establecidos en los municipios de Sibaté, finca de PROPAR S.A; Guasca, Inversiones Medina Ltda. y Subachoque, PRAJAIM S.A., en el departamento de Cundinamarca, ubicados entre 2.600-2.850msnm y temperatura promedio entre 15 a $19^{\circ} \mathrm{C}$. Se escogieron plantas 
con síntomas externos de marchitez y necrosis vasculares visibles, de las variedades Petra, entre seis y 15 meses de edad y Gold Strike, de cuatro meses.

Para el aislamiento de fitopatógenos, se utilizó el medio de cultivo papa dextrosa agar PDA, marca Merck, acidificado con 50 gotas de ácido láctico $50 \%$ por $1000 \mathrm{~mL}$ de medio (French $\mathcal{E}$ Teddy, 1986). Muestras de hojas, de raíces y de tallos, se lavaron, previamente, con agua corriente, para eliminar residuos de suelo. De cada órgano, se cortaron trozos de aproximadamente $5 \mathrm{~mm}^{2}$, los cuales, se sumergieron en agua destilada estéril durante 30 segundos, un minuto en alcohol etílico al $70 \%$, dos minutos en hipoclorito de sodio al $1 \%$ y se lavaron en agua destilada estéril por 30 segundos (French E Teddy, 1986). En cámara de flujo laminar, se sembraron cuatro trozos de tejido en cada caja de Petri, con PDA, por medio de una pinza esterilizada. Este procedimiento se realizó por triplicado, respectivamente, para cada uno de los segmentos de tejido enfermo de hojas, de raíces y de tallos, para cada una de las variedades.

Los aislamientos fueron sometidos a incubación a $25^{\circ} \mathrm{C}$ durante ocho días. Al terminar este tiempo, se hicieron observaciones sobre las siguientes características: tipo de colonia, coloración, velocidad y forma de crecimiento. La identificación microscópica del patógeno, se efectuó mediante la observación de montajes en portaobjetos, con tinción de lactofenol con azul de algodón (French E Teddy, 1986), al microscopio óptico de luz (NIKON ECLIPSE E 100), en 40X y 100X. Se observaron las características morfológicas del patógeno en cada colonia obtenida y se compararon con claves taxonómicas (Barnett, 1998). Las colonias logradas, se llevaron al Laboratorio de Micología y Fitopatología (LAMFU), de la Universidad de Los Andes, para su identificación por métodos moleculares, donde se cultivaron en medio líquido sucrosa dextrosa y extracto de levadura SDY marca Merck, durante 15 días; al cabo de este tiempo, se secó el micelio y se liofilizó. Luego del material liofilizado y seco, se extrajo el ADN, con el fin de amplificar un fragmento del ADN ribosomal, por medio de PCR. El fragmento amplificado, se secuenció utilizando BigDye ${ }^{\circledR}$ Terminator v3.1 Cycle Sequencing Kit, según las especificaciones del fabricante, en un secuenciador ABI3730XL (Applied Biosystems).

Para las pruebas de patogenicidad en laboratorio, se partió de cultivos puros de cada una de las dos cepas de Fusarium, encontradas en el experimento anterior, diferenciadas por la coloración de la colonia en PDA; una colonia blanca y otra roja, respectivamente. Para esta prueba, se usó un diseño completamente al azar con un arreglo factorial 2 × 4. Se evaluaron dos variedades, Gold Strike y Petra, bajo cuatro tratamientos. Cada variedad, se inoculó por inmersión de ramas de $18 \mathrm{~cm}$ de longitud, provenientes de plantas sanas de
Leucadendron, de seis meses de edad, en tubos de ensayo de $10 \mathrm{~cm}$, que contenían suspensiones de $1 \times 10^{5}$ macroconidias/ $\mathrm{mL}$ de agua destilada estéril. La concentración, se calculó con una cámara de Neubauer (French ETeddy, 1986). Los cuatro tratamientos fueron: Fusarium sp. (cepa roja), F. oxysporum (cepa blanca), F. sp + F. oxysporum y testigo en agua destilada esterilizada. Cada unidad experimental estuvo constituida por cinco ramas de Gold Strike y Petra, respectivamente, para un total de 60 tubos de ensayo. Las ramas inoculadas, se dejaron en laboratorio, a una temperatura promedio de $15^{\circ} \mathrm{C}$. Se hicieron observaciones cada tres días, a partir del tercer día después de la inoculación, hasta obtener cuatro lecturas. Se realizaron dos reaislamientos de ramas sintomáticas, para comprobación de patogenicidad por cada tratamiento.

Se evaluó la incidencia de la enfermedad, calculada como la proporción entre el número de plantas enfermas y el número total de plantas observadas. También, se cuantificó la severidad, medida como el número de hojas con síntomas, sobre el número total de hojas por planta (Campbell $\mathcal{E}$ Madden, 1990; Ogawa, 1986). Los datos, se sometieron a análisis de varianza ANOVA y a la prueba de rango múltiple de Tukey.

En las pruebas de patogenicidad en campo, la investigación se llevó a cabo en la unidad experimental El Remanso. En un área experimental de $60 \mathrm{~m}^{2}$ a libre exposición, se tomó una muestra de suelo, se analizó en medio peptona PCNB agar PPA (Leslie E Summerell, 2006) y se confirmó la ausencia de Fusarium spp. A continuación, se sembraron 60 plantas de la variedad Petra y 60 de la variedad Gold Strike, de tres meses de edad. El experimento, se distribuyó en cuatro camas, cada una de $9 \mathrm{~m}$ de ancho x 10,5m de largo, separadas entre sí a distancia de 1,80m. En cada cama se sembraron 30 plantas, a una distancia de $0,30 \mathrm{~m}$, entre ellas. Se instaló riego por goteo de cinta de $10 \mathrm{~cm}$, con cobertura de plástico sobre las camas. Las plantas sanas para siembra, se obtuvieron en la finca de PRAJAIM S.A., del municipio Subachoque, Cundinamarca.

Se utilizó un diseño de bloques completamente al azar, con arreglo factorial $2 \times 4$. Se tuvieron en cuenta las dos variedades como factor: Gold Strike y Petra. Se hicieron cuatro tratamientos con tres repeticiones, para un total de doce unidades experimentales, cada una constituida por cinco plantas. Los tratamientos fueron: Fusarium sp. (cepa roja), F. oxysporum (cepa blanca) y $F$. sp $+F$. oxysporum y agua destilada estéril. La inoculación consistió en la inyección, en el cuello de la planta, de $3 \mathrm{~mL}$ de una suspensión de $10^{5} \mathrm{~mL}^{-1}$ macroconidias, calculadas con el empleo de una cámara de Neubauer (French \& Teddy, 1986). Para facilitar la operación, se produjo una herida de $1 \mathrm{~cm}$ de largo en el cuello de la raíz, con bisturí, previamente sumergido en alcohol al 70\%; las plantas testigo, se inocularon con agua destilada estéril. Se 
evaluó la incidencia de la enfermedad durante 12 semanas (Campbell E Madden, 1990; Ogawa, 1986). Para cuantificar la severidad, mediante un muestreo destructivo, se midió, con una regla, la longitud de la lesión vascular, a partir del cuello de la planta hasta el primer nudo, donde se bifurcan las ramas secundarias. Se hicieron re-aislamientos en PDA por cada tratamiento, para comprobar la presencia del fitopatógeno inoculado. Con los datos obtenidos de severidad, se elaboró el análisis de varianza y la prueba de rango múltiple de Tukey.

\section{RESULTADOS Y DISCUSIÓN}

Los síntomas de la marchitez, se manifiestan en hojas, en tallo y en raíces. En las hojas, se caracterizan por la presencia de clorosis, que se inicia en el ápice y bordes y avanza, progresivamente, hacia el centro, hasta cubrir toda la hoja. Esta sintomatología coincide con la descripción manifestada por Lubbe et al. (2008), Moura E Rodrigues, (2001), Herrera et al. (2006). En la medida en que la enfermedad progresa, se presenta necrosis en las áreas afectadas. En los tallos, se evidencia pérdida de coloración normal y brillo; se tornan oscuros en toda su longitud y, cuando se hace un corte longitudinal, se observa el tejido vascular manchado de color marrón (Figura 1). En el cuello de las raíces, se exterioriza una pudrición seca, que se extiende por los tejidos de la epidermis, forma una masa corchosa granular, que se deshace fácilmente, cuando se hace disección. Estos síntomas concuerdan con los descritos por Lubbe et al. (2008) y Crous et al. (2004), para $F$. oxysporum. En las muestras evaluadas, no se detectaron signos del fitopatógeno.

En los aislamientos realizados de tejidos sintomáticos de marchitez vascular, en las variedades Petra y Gold Strike, prevaleció la presencia de Fusarium spp. Dos cepas diferentes de este fitopatógeno fueron recurrentes: una colonia de color blanco y otra de pigmentación roja. La primera, se caracterizó porque su coloración en su estado inicial es rosada y a medida que envejece toma un color blanco con variantes rosadas. Es de textura algodonosa y produce pigmento de color rosado grisáceo, que se observa en la base del medio de cultivo. Las colonias son de crecimiento rápido y se extienden en forma radial. A los tres días, después del aislamiento, alcanza un diámetro de $2 \mathrm{~cm} \mathrm{y}$, a los ocho días, el micelio cubre toda la superficie del medio.

La colonia roja de Fusarium es inicialmente amarilla, luego cambia a naranja, con variantes rojas y, posteriormente, adquiere un color rojo profundo, igual al pigmento que se observa en la base del medio de cultivo. Esta cepa tarda un poco más en crecer. Su expansión en el medio es radial y a los siete días, después del aislamiento, la colonia alcanza un diámetro variable entre 4 y $6 \mathrm{~cm}$. En 15 días, el micelio cubre toda la superficie del medio PDA.
Las apariencias morfológicas generales y coloraciones de las colonias son parámetros de diagnóstico útiles en la identificación de especies (Booth, 1971; Nelson et al. 1994; Leslie E Summerell, 2006); sin embargo, la pigmentación de las cepas es, además, una respuesta a la interacción entre el medio de cultivo, el hongo y factores físicos y químicos, como luz, pH y temperatura de incubación (Leslie $\mathcal{E}$ Summerell, 2006). En la especie $F$. oxysporum, la morfología de la colonia en PDA es muy variable, presenta diferencias en color, en consistencia del micelio y presencia de conidias (Nelson et al. 1994). El micelio puede ser escaso o abundante y la variación en color comprende una gama que va desde el blanco al violeta pálido. Con masas de macroconidias naranja a violeta o la producción de pigmentos en el medio, en tonos variables entre violeta y magenta, produciendo una masa de conidias en el centro en algunos aislamientos (Leslie E Summerell, 2006).

Los resultados anteriores sobre la presencia de dos cepas diferentes del género Fusarium asociadas con la marchitez en proteas, coincide con los resultados de la caracterización molecular, realizada en el Laboratorio de Micología y Fitopatología (LAMFU), de la Universidad de los Andes. La cepa blanca corresponde a F. oxysporum, con un $99 \%$ de certeza. La cepa roja no fue plenamente definida y se sospecha que el aislamiento corresponde a un Fusarium cf. graminearum, posiblemente, Fusarium vorosii o Fusarium aethiopicum. De acuerdo a estos resultados, la segunda cepa de color rojo, se denominó como Fusarium sp. En consecuencia, se tiene un vacío en la identificación y se recomienda utilizar el gen de elongación alfa sobre varias cepas del fitopatógeno, con el fin de obtener la especie exacta (Vázquez E Berron, 2004; Leslie E Summerell, 2006; O'Donnell et al. 2008). En la presente investigación, no se hicieron estudios adicionales para la designación de formae specialis y de razas, que se derivan de la relación fitopatológica entre este fitopatógeno y diferentes cultivos, incluyendo proteas. De acuerdo a Crous et al. (2004), no se han denominado formae specialis de este fitopatógeno para proteas y, por lo tanto, se deben adelantar estudios de este tipo, puesto que cepas de F. oxysporum tienen un alto grado de especificidad hacia sus hospedantes y dan origen a subdivisiones taxonómicas (Leslie E Summerell, 2006).

En las pruebas de patogenicidad en laboratorio, se observaron síntomas de marchitez, sobre las variedades Petra y Gold Strike, a partir del tercer día después de inoculación (DDI), lo que indica que existe una interacción compatible entre los hongos fitopatógenos aislados e inoculados y la manifestación de la enfermedad. En los testigos no inoculados, no se observaron síntomas de enfermedad. Al tercer DDI hubo una incidencia del $80 \%$ en la variedad Gold Strike, con Fusarium sp., y $F$.oxysporum + Fusarium sp.; y, del 40\%, cuando se inoculó sólo $F$. oxysporum. Caso contrario en la variedad Petra, donde la incidencia fue de $60 \%$, con F. oxysporum y 

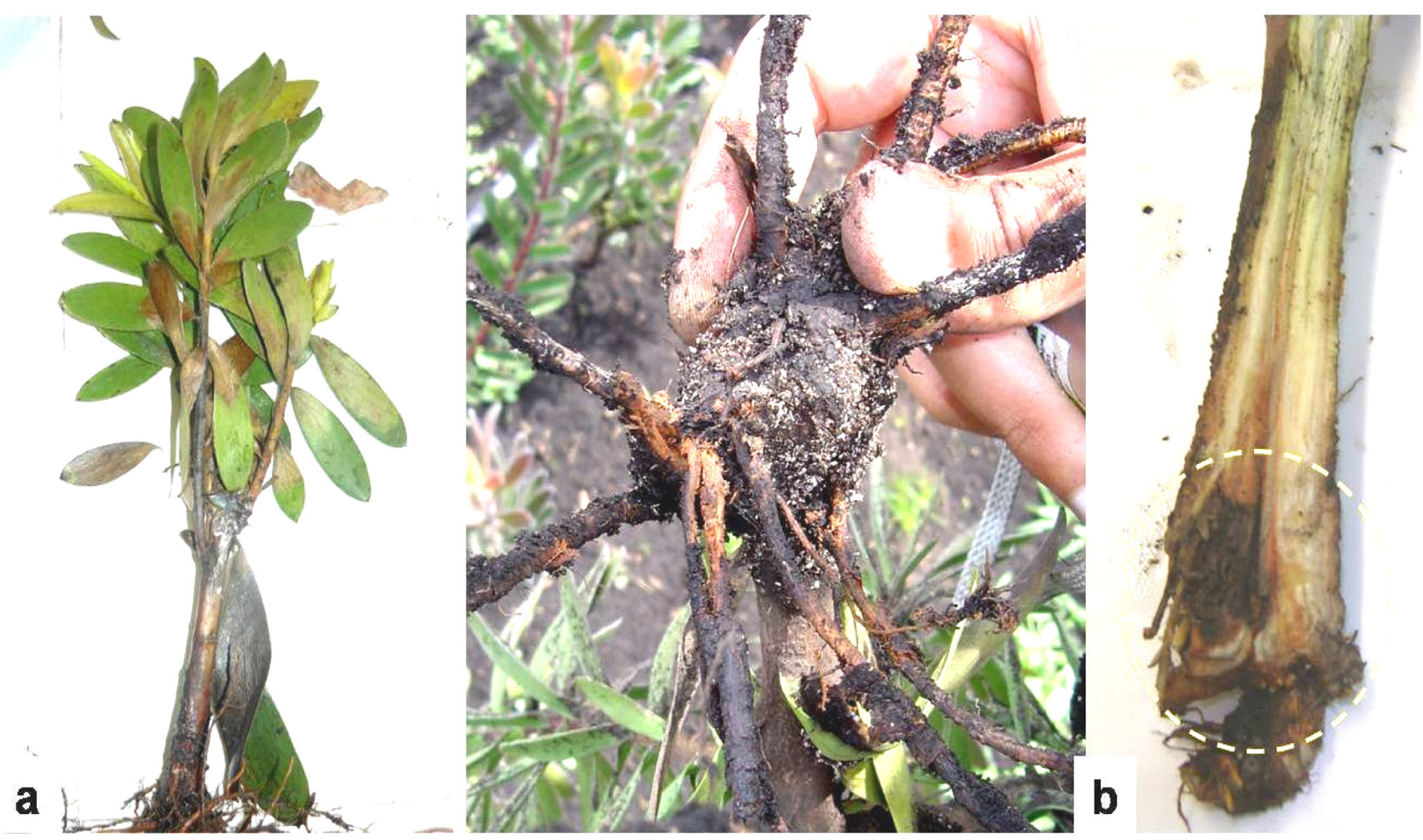

Figura 1. Síntomas de marchitez en proteas variedad Gold Strike. a) Parte aérea de la planta; b) Pudrición seca de raíces y cuello de la planta.

Fusarium sp. y, del 40\%, cuando se inoculó con la mezcla $F$. oxysporum + Fusarium sp.

En estos primeros días, se observaron diferencias entre las interacciones variedad - patógeno, que explican la existencia de niveles de especificidad en los procesos iniciales de infección y de colonización, los cuales, dependen de la variación existente dentro de la población de macroconidias y de las condiciones de temperatura y de humedad existentes (Campbell \& Madden, 1990); sin embargo, estas diferencias se redujeron a través del tiempo y a los nueve DDI, los tres tratamientos llegaron al $100 \%$ de incidencia en las variedades estudiadas (Figura 2).

En la primera evaluación de severidad a los tres DDI, se presentaron diferencias altamente significativas $(P<0,0001)$ entre los tratamientos, dentro de cada variedad. En la variedad Petra, F. oxysporum tuvo la mayor proporción de hojas afectadas, con 18\%; siguió la mezcla F. oxysporum+ Fusarium sp., con 9\% y Fusarium sp., también con $9 \%$ (Figura 3). Resultado opuesto se encontró en la variedad Gold Strike, donde Fusarium sp. y la mezcla dieron los valores más altos, con 38 y $36 \%$, respectivamente, en comparación con $F$. oxysporum, que produjo una severidad de $4 \%$. Estos resultados indican que $F$. oxysporum tuvo mayor capacidad para colonizar tejidos y generar el bloqueo de vasos en la variedad Petra. Por su parte, la marchitez por Fusarium sp. fue más severa en Gold Strike. Aunque la mezcla de las dos cepas también produjo infección, ésta fue mayor en Gold Strike que en Petra. Este resultado podría indicar la existencia de algún grado de antagonismo entre las especies de Fusarium, cuando interactúan con la variedad Petra.

Todos los tratamientos con Fusarium fueron significativamente diferentes con respecto al testigo no inoculado $(P<0,001)$. La tendencia de las dos variedades frente a $F$. oxysporum se mantiene en el noveno día: Petra tuvo mayor porcentaje de severidad (90\%) que Gold Strike $(40 \%)$. Con relación a Fusarium sp., Petra tuvo menor valor (71\%) que Gold Strike (85\%). Respecto a la mezcla de las dos cepas, Petra alcanzó 95\% y Gold Strike 76\%. Esto explica que la variedad Gold Strike es más tolerante a $F$. oxysporum y a la mezcla de las dos especies; caso contrario en la variedad Petra, la cual, tiende a ser mas susceptible. 


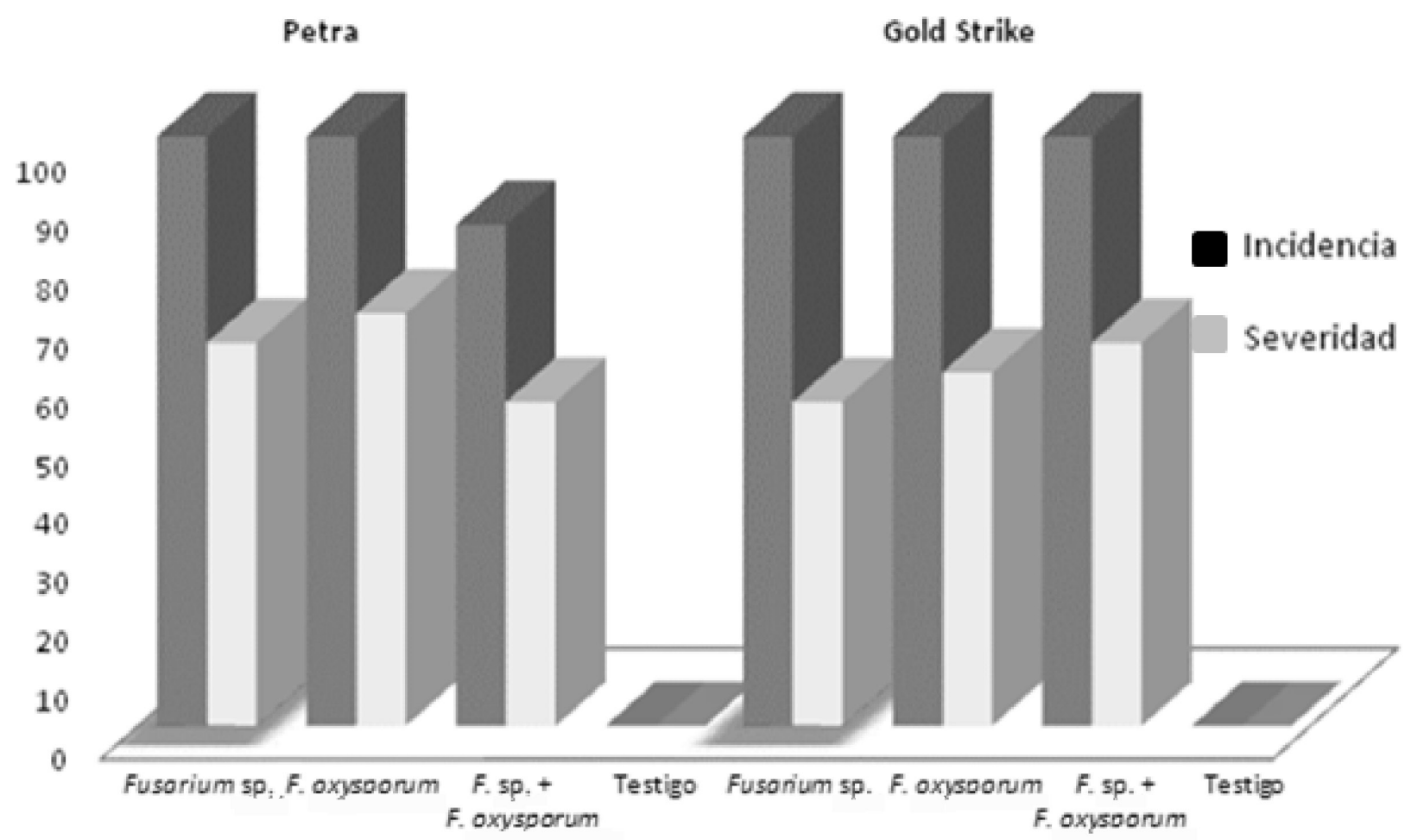

Figura 2. Porcentaje de incidencia y de severidad de marchitez en proteas variedades Petra y Gold Strike, 15 días después de inoculación, con las dos especies de Fusarium en condiciones de laboratorio.

En las pruebas de patogenicidad en campo, la incidencia de la marchitez a las 12 semanas fue variable para los diferentes tratamientos: $13 \%$, para Fusarium sp. y, $7 \%$, para F. oxysporum + Fusarium sp. Las plantas inoculadas con F. oxysporum y las plantas testigo no manifestaron ninguna sintomatología. En las plantas sintomáticas, la aparición de la marchitez se inició con pérdida de turgencia de la parte apical de las ramas principales de la planta, causando la declinación de sus brotes. Posteriormente, se presentó marchitamiento en las hojas basales del tallo principal, el cual, progresa hacia la parte superior, extendiéndose en todo el follaje de la planta. Sus hojas permanecieron rígidas y adheridas al tallo de la planta. En el muestreo destructivo, se percibió una lesión vascular de color marrón y tonalidades rojizas en la zona de los vasos conductores del xilema, que se atenúa en el cuello de las raíces. A partir de esta región, la lesión continúa a lo largo del tallo en forma ascendente, hasta el primer nudo, donde se bifurcan las ramas secundarias.

El avance de la lesión depende de la severidad de la enfermedad (Agrios, 2005) y la coloración, se debe a la oxidación y a la obstrucción de los vasos del xilema. Los cortes transversales en tallos infectados con las dos especies de Fusarium muestran varias zonas cafés, pronunciadas y decoloradas, dispuestas en forma de anillo completo o interrumpido, en el área de los haces vasculares, cerca de la base de la planta. En las raíces no se presentaron lesiones internas, pero el volumen de raíces era bajo en plantas enfermas, cuando se comparan con plantas testigo sin inocular.

La demora en la manifestación de síntomas externos de la enfermedad en el follaje explica que la colonización avanza lentamente en los tejidos conductores y confirma la sintomatología de ataques por $F$. oxysporum, en plantas leñosas (Roncero et al. 2003; Crous et al. 2004). Según Dickinson (1987), los agentes causantes de los marchitamientos vasculares pueden ocasionar la muerte de plantas en pocas semanas, pero en algunas ocasiones, tarda más en presentar los síntomas, dependiendo del tipo de hospedante y de su tejido vegetal. En este caso, las plantas del género Leucadendron están formadas por tejido leñoso y se caracterizan por ser perennes. Algunas de estas plantas presentan síntomas de marchitez o muerte después de varios años, a partir del momento que fueron infectadas por el hongo (Obreque, 2004; Swart et al. 2001; Crous et al. 2004). 


\section{Petra}

\section{Gold Strike}

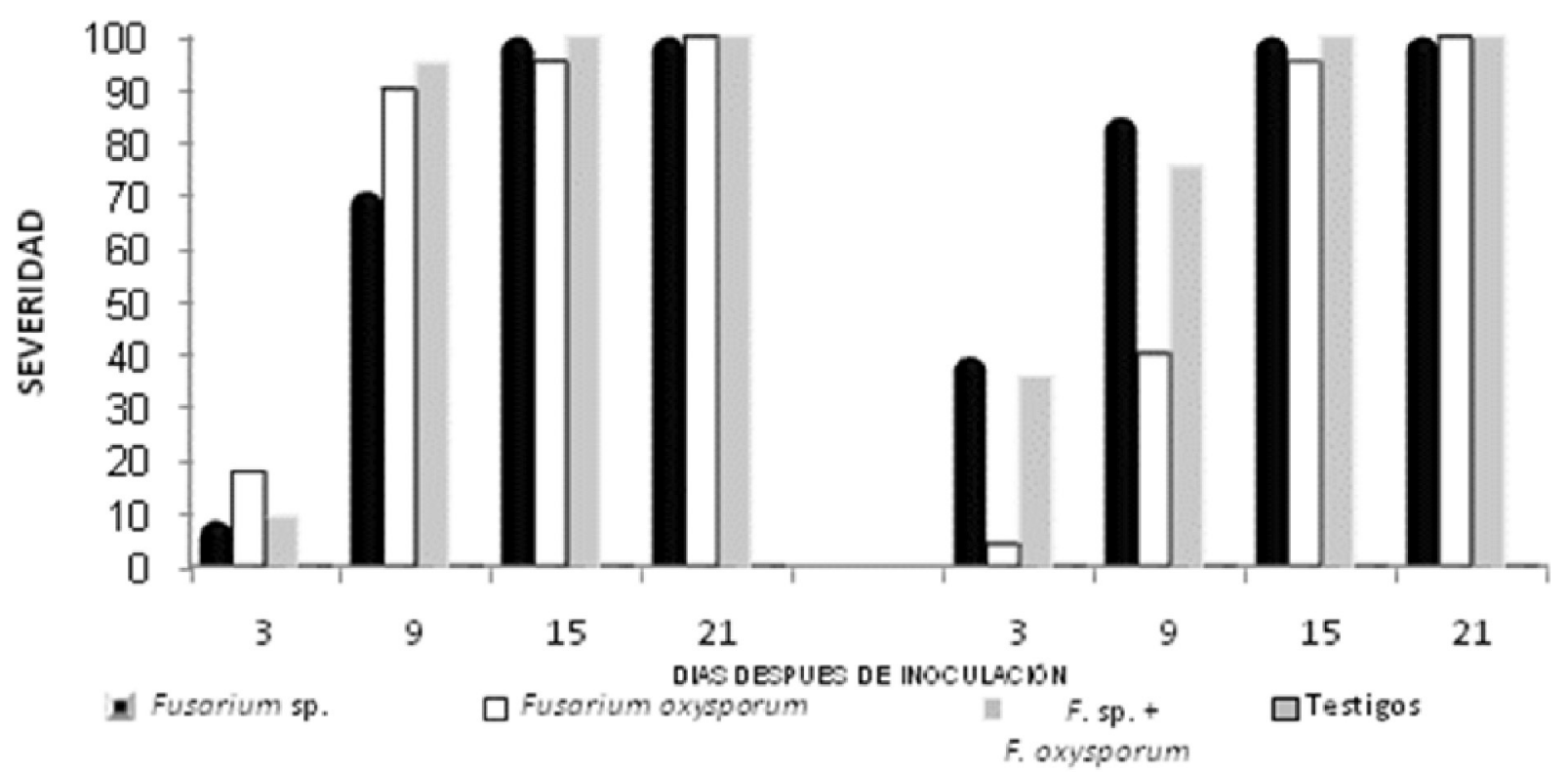

Figura 3. Porcentaje de severidad de marchitez en proteas variedades Petra y Gold Strike a los 3, 9, 15 y 21 dias después de inoculacion, con las dos especies de Fusarium, en condiciones de laboratorio.

Por consiguiente, el patógeno continúa propagándose internamente en forma de micelio o conidias, a través de los vasos del xilema, hasta que muere toda la planta. Este tejido leñoso también es una barrera que impide al fitopatógeno la penetración de paredes y de membranas celulares e invadir vascularmente, ya que sus tejidos son más rígidos y dificultan el proceso de colonización. Esto hace parte de la interacción celular y molecular entre el hospedante y las correspondientes formae specialis y razas del patógeno (Roncero \& Di Prieto, 2005). Las plantas adultas, se pueden marchitar y morir repentinamente, cuando una infección es severa y el clima favorece el desarrollo de la enfermedad. Bajo estas condiciones, se puede incrementar la velocidad de la epidemia y la manifestación de síntomas (Agrios, 2005).

En la prueba de campo, la incidencia de la enfermedad fue variable a nivel vascular. El $100 \%$, se encontró en las plantas inoculadas con $F$. oxysporum y Fusarium sp., respectivamente, en las dos variedades y, el $80 \%$, cuando las plantas se inocularon con la mezcla F. oxysporum + Fusarium sp., en la variedad Petra (Figura 4).

Hubo diferencia altamente significativa en severidad $(\mathrm{F}<$ $0,001)$ entre los tratamientos evaluados en las dos variedades
Petra y Gold Strike y el testigo, pero no se registraron diferencias estadísticas entre el grado de severidad de las inoculaciones con Fusarium sp., F. oxysporum y la mezcla F. oxysporum + Fusarium sp. Estos resultados comprueban que $F$. oxysporum y Fusarium sp. son patógenos que causan la marchitez. De manera individual y en mezcla, tuvieron la misma capacidad para colonizar el tejido vascular de plantas en las dos variedades, lo cual, implica que los productores de proteas se enfrentan a dos patógenos que ponen en riesgo su producción y su rentabilidad. Se debe resaltar que $F$. oxysporum es un fitopatógeno de alta patogenicidad que ataca diferentes especies vegetales. Además, su control es imposible, una vez establecido en el campo y, en consecuencia, se deben aplicar medidas de sanidad vegetal dirigidas a reducir su diseminación. Con respecto a Fusarium sp., se requiere profundizar en el conocimiento de su biología, en su sistemática y su control.

Con base en las pruebas de patogenicidad realizadas en condiciones de laboratorio y de campo, se pudo establecer el registro de la marchitez, como una nueva patología en cultivos de proteas en Colombia, asociada a la incidencia de dos especies del género Fusarium: F. oxysporum y Fusarium sp. Estos fitopatógenos son sistémicos vasculares 


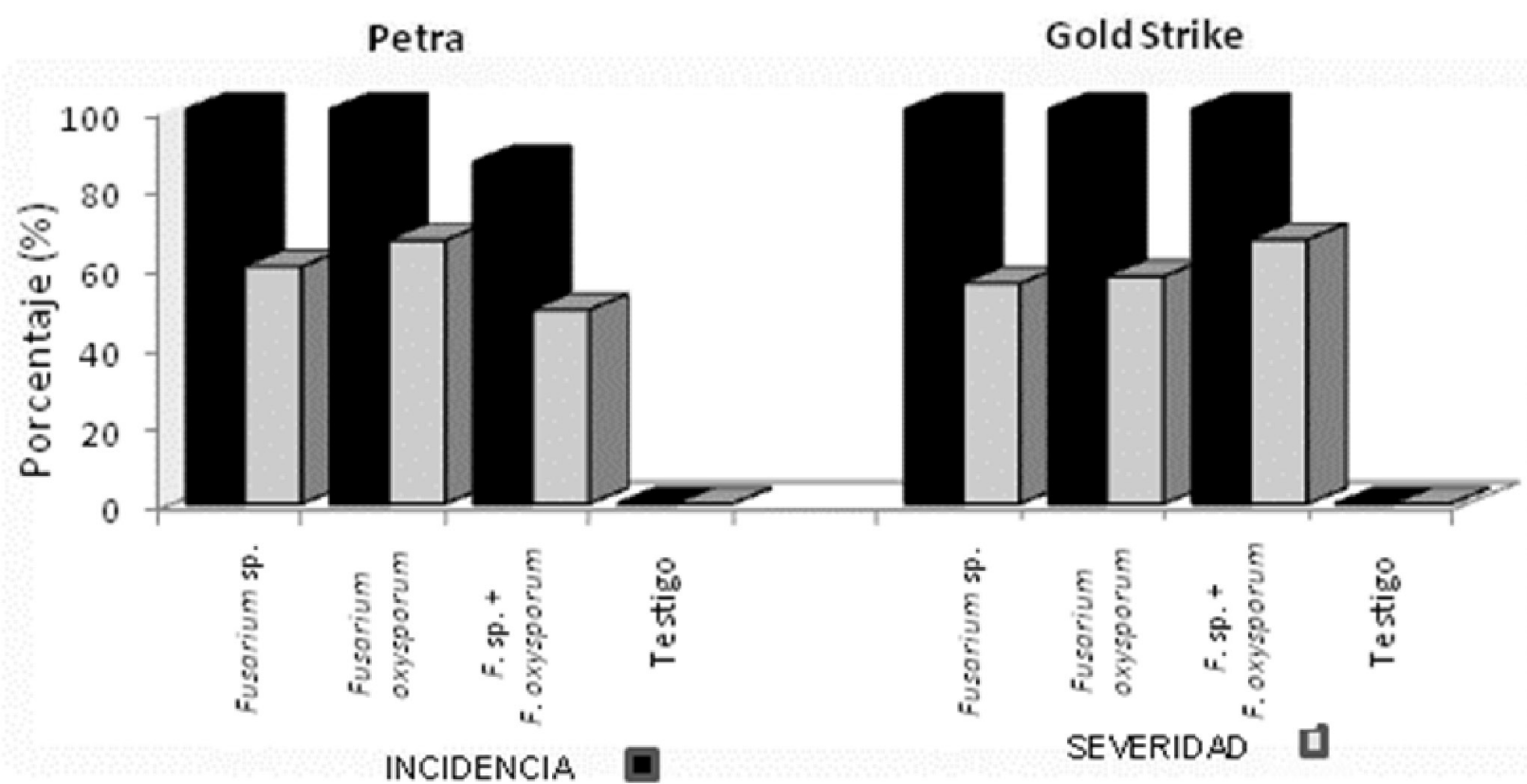

Figura 4. Incidencia y severidad de marchitez en proteas variedades Petra y Gold Strike, doce semanas después de inoculación, con las dos especies de Fusarium, en condiciones de campo.

y se pueden diseminar por medio del material vegetal de propagación de proteas; por tanto, representan un alto riesgo para la producción comercial en el país, que se sustenta en la importación de semilla vegetativa. Sería conveniente buscar alternativas que garanticen un material de excelente calidad fitosanitaria.

Conflictos de interés: El manuscrito fue preparado y revisado con la participación de todos los autores, quienes declaramos que no existe ningún conflicto de intereses que ponga en riesgo la validez de los resultados presentados. Financiación: Este estudio contó con la financiación parcial de la Universidad de Ciencias Aplicadas y Ambientales U.D.C.A.

\section{BIBLIOGRAFÍA}

1. AGRIOS, G.N. 2005. Plant Pathology. 5th Edition. Elsevier Academic Press. USA. 922p.

2. BARKERA, N.P.; VANDERPOORTENB, A.; MORTONC. C.M.; ROURKE, J.P. 2004. Phylogeny, biogeography, and the evolution of life-history traits in Leucadendron (Proteaceae). Molecular Phylogenetics and Evolution. 33:845-860.

3. BARNETT, L.H. 1998. Illustrated genera of imperfect fungi. The American Phytopathological Society. St. Paul, Minesota, USA. 218p.
4. BEN, J. 1986. Technology for rapid production of flowering pot- plants of Leucadendron discolor. Scientia Hort. 28:397-388.

5. BOOTH, C. 1971. The genus Fusarium. The present status of Fusarium taxonomy. Ferry Lane, Kew y Surrey. Inglaterra. Commonwealth Mycological Institute, 58p.

6. CAMPBELL, C.; MADDEN, L. 1990. Introduction to plant disease epidemiology. John Wiley \& Sons. 532p.

7. CROUS, P.W.; PALM. M.E. 1999. Systematics of selected foliicolous fungi associated with leaf spots of Proteaceae. Mycol. Res. 103(10):1299-1304.

8. CROUS, P.W.; DENMAN, S.; TAYLOR, J.E.; SWART, L.; PALM, M.E. 2004. Cultivation and Diseases of Proteaceae: Leucadendron, Leucospermum and Protea. CBS, Biodiversity Series. 2:1-228.

9. DENMAN, S. 2001. Evaluation of a stem inoculation technique for assessing resistance to Phytophhora cinnamomi in Leucadendron y Leucospermun cultivars. Acta Hort. 545:281-285.

10. DICKINSON, C.H. 1987. Patología vegetal y patógena de plantas. Noriega Editores. Editorial Limusa S.A. México. p.11-179. 
11. DUNNE, C.; DELL, B.; HARDY, G. 2003. "Sudden death" in proteas in the southwest of western Australia. Acta Hort. (ISHS) 602:39-44.

12. FRENCH, R.; TEDDY, T. 1986. Métodos de Investigación Fitopatológica. Capítulo 10. Aislamiento Fitopatógenos. Costa Rica. p.158-161.

13. HERRERA, R.; OBREQUE, M.; VICO, V.; REBOLLEDO, P.; LOLAS, M.; SCHIAPPACASSE, F.; SANDOVAL, C. 2006. Fungi and bacteria affecting protea plantings in Chile. Acta Hort. (ISHS) 716:107-110.

14. INTERNATIONAL SOCIETY FOR HORTICULTURAL SCIENCE (ISHS). 2007. Proteaceous Ornamentals: Banksia, Leucadendron, Leucospermum, and Protea. Reprinted with permission of John Wiley \& Sons, Inc., from Horticultural Reviews $\mathrm{N}^{\circ}$ 5. Gent-Oostakker, Belgium. 160p.

15. LESLIE, J.F.; SUMMERELL, B.A. 2006. The Fusarium Laboratory Manual. Blackwell Publishing, Ames, lowa, $388 p$.

16. LUBBE, C.M.; LAMPRECHT, S.C.; VAN NIEKERK, J.M.; MOSTERT, L. 2008. Molecular characterization of Fusarium oxysporum causing wilt of proteaceae. Acta Hort. (ISHS) 805:127-134.

17. MATTHEWS, L. 2002. The protea book. A guide to cultivated Proteaceae. New Zealand. Canterbury University Press. 546p.

18. MALAN, D. 1992. Propagation of Proteaceae. Acta Hort. 316:27-34.

19. MOURA, M.F.; RODRIGUES, P.F. 2001. Fungal diseases on proteas identified in madeira island. Acta Hort. (ISHS) 545:265-268.

20. NELSON, P.; DIGNANI, C.; ELIAS, A. 1994. Taxonomy, biology, and clinical aspects of Fusarium Species. Clin. Microbiol. Rev. 7(4):479-504.

21. OBREQUE, X. 2004. Evaluación de aplicaciones preinfección del fungicida Benomilo y del biocontrolador Trichoderma harzianum en el control de Fusarium sp. en Proteaceas. Disponible desde internet en http:// dspace.utalca.cl/retrieve/2970/MObrequeD.pdf (con acceso el 25/06/09).
22. O’DONNELL, K.; WARD, T.J.; ABERRA, D.; KISTLER, H.C.; AOKI, T.; ORWING, N. 2008. Multilocus genotyping and molecular phylogenetics resolve a novel head blight pathogen within the Fusarium graminearum species complex from Etiopía. Fungal Genet. Biol. 45(11):1514-1522.

23. OGAWA, J. 1986. Fields test procedures for evaluation of fungicides to control. In: Hickey, K. (Ed.). Methods for evaluating pesticides for control of plants pathogens. Am. Phytophat. Soc. Press.p.152.-154.

24. RABELO, T. 1995. A field guide to the proteas of the southern Africa. National Botanical Institute. Sudafrica. 345p.

25. RONCERO, M.; DI PIETRO, A. 2005. Papel de enzimas líticas de la pared celular en la patogenicidad de Fusarium oxysporum. Rev. Iberoam. Micología (Venezuela) 17:S47-S53.

26. RONCERO, M.I.G.; HERA, C.; RUIZ-RUBIO, M.; GARCÍA MACEIRA, F.I.; MADRID, M.P.; CARACUEL, Z.; CALERO, F.; DELGADO-JARANA, J.; ROLDÁN-RODRÍGUEZ, R.; VELASCO, C.; ROA J.; DI PIETRO, A. 2003. Fusarium as a model for studying virulence in soilborne plant pathogens. Physiol. Mol. Plant Path. 62:87-98.

27. SALINGER, P. 1991. Producción comercial de proteas. Especies, híbridos. $3^{\mathrm{a}}$ Ed. Zaragosa, Scriba. 371p.

28. SWART, L.; DEMMAN, S.; LAMPRECHT, P.; CROUS, P. 2001. Fusarium wilt: A new disease of cultivated Protea in South Africa. Australasian Plant Path. 258(2):156161.

29. TAYLOR, J.E.; CROUS, P.W. 2000. Fungi occurring on Proteaceae: new anamorphs for Teratosphaeria, Mycosphaerella and Lembosia, and other fungi associated with leaf spots and cankers of proteaceous hosts. Mycol. Res. 104(5):618-636.

30. VÁZQUEZ, J.; BERRON, S. 2004. Multicolus MLST. Sequence typing: el marcador molecular. Laboratorio de referencia de Neisserias. Centro Nacional de Microbiología. Instituto de salud Carlos III. Majadahonda. Madrid. España. Enferm. Infecc. Microbiol Clin. 22(2):113-120.

Recibido: Mayo 31 de 2010

Aceptado: Noviembre 5 de 2010 